\title{
Dynamical effects in fission investigated at high excitation energy
}

\author{
J. Benlliure
}

Universidad de Santiago de Compostela, E-15782 Santiago de Compostela, Spain

\begin{abstract}
The experimental techniques used for the investigation of nuclear fission have progressed considerably during the last decade. Most of this progress is based on the use of the inverse kinematics technique allowing for the first time the complete isotopic and kinematic characterization of both fission fragments. These measurements make possible to characterize the fissioning system at saddle and at scission, and can be used to benchmark fission model calculations. One of the important ingredients in transport models describing the dynamics of the process is the dissipation parameter, governing the coupling between intrinsic and collective degrees of freedom. Recent experiments got access to the magnitude of this parameter and could also investigate its dependence in temperature and deformation.
\end{abstract}

\section{Introduction}

More than 75 years after its discovery, fission still represents one of the most fascinating processes in nuclear physics, but also an important challenge for physicists. Indeed, nuclear fission has implications in many different domains such as the investigation of shell effects at large deformation [1] or the dynamics of highly excited nuclear matter [2], but also in the understanding of the abundances of elements produced in the stellar nucleosynthesis $\mathrm{r}$ process [3]. Moreover, fission also has a relevant societal impact because of its role in energy generation and radioisotope production for medical imaging. 
Despite the fact that a global understanding of nuclear fission was proposed soon after its discovery [4], we are still far from a complete microscopic description. The reason being that fission is a conceptually complicated process where collective and single-particle degrees of freedom interplay. The first attempt to describe the dynamics of fission was put forward by Kramers [5] assuming that the evolution of the deformation degree of freedom can be viewed as a Brownian motion of this collective degree of freedom with a heat bath defined by the single-particle degrees of freedom. The timedependent formulation of this model using a Fokker-Planck equation with a dissipative term describing the coupling between the collective and singleparticle degrees of freedom was introduced by Grangé and collaborators [6]. An analogue formulation is the one based on the Langevin equation [2] or as a random walk, in the limit of strong dissipative coupling [7], on a multidimensional macroscopic-microscopic potential energy surface [8].

Accurate microscopic descriptions of the potential energy surface are nowadays obtained with modern implementations of the density functional theory [9]. However, a microscopic description of the full fission dynamics still represents a challenge for theorists. In this respect, approaches based on the time-dependent Hartree-Fock theory have provided relevant progress in the description of the fission dynamics by including nonadiabatic effects close to the scission point [10].

From an experimental viewpoint, the complete characterization of the fission fragments has been a challenge because of the low energy and high $\mathrm{Z}$ values of the heavy fission fragments. The use of inverse kinematics and state-of-the-art detection setups made possible to overcome this situation very recently. On another hand, the dynamics of the fission process has been mostly addressed by using low-energy fission reactions. However, it has been demonstrated that transient effects governing the ground-to-saddle dynamics only manifest in fast fission reactions induced at high energies. In this work we will briefly review the main advances in the investigation of the fission dynamics and we will then concentrate of recent results concerning these investigations at high excitation energy.

\section{Investigation of dynamical effects in fission}

The time scale and energy dissipation during the fission path from saddle to scission have been investigated at low energies using observables giving access to the the scission-point configuration. For example, total kinetic energies of the fragments give access to the elongation at scission of the fis- 
sioning system [11] The sawtoothlike behavior of the prompt-neutron yields was generally attributed to the contribution of the deformation energy to the excitation energy of the fragments [12]. Finally, the odd-even effect in fission-fragment element distributions is another observable used to characterize the energy dissipated between saddle and scission in low-energy fission [13]. However, the interpretation of some of these observable has been recently revisited. K.-H. Schmidt and collaborators explain the sawtoohlike pattern of the prompt-neutron yields, and in particular its energy dependence, and the general trends observed in the odd-even staggering, as due to the energy sorting between both fission fragments according to their size $[14,15]$.

The ground-to-saddle dynamics can be investigated using observables sensitive to the corresponding time. The emission of prescission gamma rays and particles, fission cross sections and the width of the charge distribution of the fission fragments have shown that typical ground-to-saddle times are longer than expected from pure statistical considerations [16]. This delay of the fission time is understood as the transient time needed for the coupling between intrinsic and collective degrees of freedom until a stationary flow of the fission width over the barrier is reached. That time can be quantified in terms of a nuclear viscosity parameter in transport equations [6]. Most of the measurements are compatible with a value of the transient time around $1-510^{-21} \mathrm{~s}$. Those values are negligible when compared to typical statistical times for low-energy fission, therefore reactions inducing fission at high-excitation energies are better suited [17]. Indeed, Grangé and collaborators [6] established that the best conditions for the manifestation of transient effects are the use of reactions leading to fissioning systems not only with large values of excitation energy but also with low values of angular momentum and spherical shapes.

\section{Experimental approaches}

Most of the information we have on the saddle-to-scission dynamics at low energy was obtained using neutron-induced fission [12]. This approach allows a precise definition of the initial compound nuclei but can only be applied with a limited number of fissionable target materials. This limitation was overcome using fusion-fission reactions [18] or beta-delayed fission [19]. However, these reactions do not allow for a complete identification of the fission fragments because the charge and velocity of the heavy-fission fragments do not allow to correlate any energy loss with their atomic number. 
The real step forward in the investigation of fission was brought by the use of the inverse kinematics. Under those conditions, the boosted fission fragments can be fully identified in atomic and mass number from magnetic rigidity, time of flight and energy-loss measurements [20]. Moreover, one can take advantage of the secondary beams produced in-flight using fragmentation reactions to investigate Coulomb-induced fission for a large number of actinides and pre-actinides [21]. This technique has been also used to investigate the saddle-to-scission dynamics in transfer-induced fission reactions [22]. However, the complete identification of both fission fragments was just achieved recently by combining the inverse kinematics with a large acceptance detection setup [23].

The inverse kinematics also allows to induce fission using fragmentation or spallation reactions [24-29]. Those reactions produce fissioning nuclei with large values of excitation energy giving access to the short time delay induced by dissipation until the fission flow over the barrier reaches a stationary regime. Moreover, spallation and fragmentation reactions introduce small shape distortions and low angular momenta. Therefore, these reactions are considered as an optimum tool for the investigation of the ground-to-saddle dynamics [30,31].

\section{Ground-to-saddle dynamics}

The investigation of the dynamics between the initial state of the fissioning compound nucleus and its configuration at saddle should provide relevant information on the energy transfer between intrinsic and collective degrees of freedom in fission that can be quantified in terms of a dissipation parameter [6]. Those investigations have been mostly carried out using fusionfission reactions $[17,32-38]$. However, those works provided rather unclear conclusions concerning the absolute value of the dissipation parameter and its possible dependences on temperature and deformation. Some reasons could be the strong influence of angular momentum and deformation in the fission probability, and the limited values of excitation energies reached in these reactions $[30,31,39]$.

An interesting alternative to fusion-fission is the use of spallation reactions induced by energetic protons [40,41] or anti-protons [42], and fragmentation reactions $[30,43]$. These reactions are expected to fulfill the conditions for the manifestation of dissipative effects in fission [6] because they can produced fissioning nuclei with large values of excitation energy but low angular momenta. Moreover, the investigation of these reactions in inverse kinemat- 
ics facilitates the identification of the fission fragments, giving access to new observables to better constraint dissipative effects in fission [44].

The ground-to-saddle time, characterizing the fission dynamics at small deformation, can only be deduced from indirect measurements. Some of those are based on measurements of the multiplicity of light-charged particles emitted pre-scission [45]. Unfortunately, those measurements do not only depend on the presaddle transient time but also on the saddle-to-scission time. Fission probabilities $[33,37,39,46,47]$ or the complementary cross sections of evaporation residues $[36,41,48]$ seem to be better suited to constraint presaddle effects. However, fission or evaporation residue cross sections depend on factors other than dissipation such as the fission barriers or the level densities $[37,49,50]$. Indeed, some authors claim that cross sections of fission reactions induced at relatively high energies can be described using purely statistical models, provided that level densities at saddle and ground configuration are adjusted to specific values $[51,52]$.

In order to overcome this confusing situation it was proposed the combined measurement of several independent observables constraining the main parameters defining the fission probability. The first observable proposed, together with the fission cross sections, was the width of the charge distribution of the final fission fragments [40]. The choice of this observable was based on the fact that the width of the charge or mass distribution of the fission fragments at their initial formation close to the saddle point is determined by statistical fluctuations and therefore by temperature [53]. Moreover, if one takes into account that fission fragments deexcite mainly emitting neutrons, then we conclude that the charge distribution of the final fragments should be very close to the same distribution at saddle and should provide an indirect measurement of the energy dissipated between ground and saddle.

A step further was proposed in Refs. [43,44] where the charge distribution of the final fission fragments was sorted as a function of the sum of the atomic numbers of the two fragments. In this case, the sum of the atomic numbers of the two fragments represents the size of the fissioning nuclei. In spallation and fragmentation reactions the size of the compound nuclei is correlated with the impact parameter and then, with the energy gained during the collision phase. In these works it was also proposed to correlate the fission cross sections with the size of the fissioning nucleus. In Fig. 1 we depict an example of both graphs obtained for the reaction ${ }^{208} \mathrm{~Pb}+\mathrm{p}$ at $500 \mathrm{~A} \mathrm{MeV}$ taken from [54]. As can be seen in the upper panel, fission cross sections 

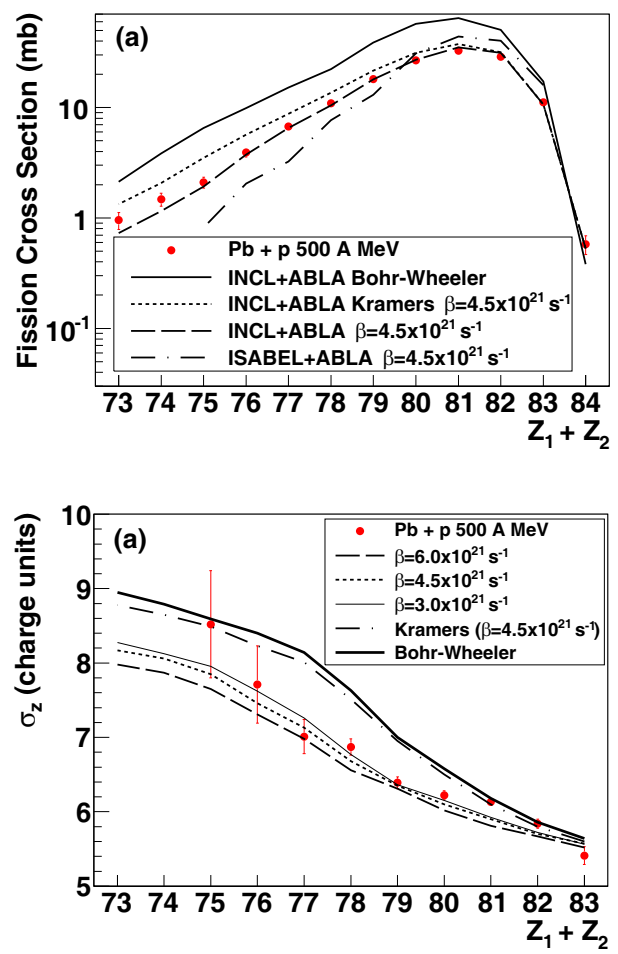

Figure 1: Upper panel: partial fission cross sections (dots) as a function of the size of the fissioning nuclei obtained for the reaction ${ }^{208} \mathrm{~Pb}+\mathrm{p}$ at $500 \mathrm{~A} \mathrm{MeV}$ compared to different model calculations performed with the intranuclear cascade codes INCL and ISABEL coupled to deexcitation code ABLA. Lower panel: correlation between the width of the charge distribution of the fission fragments and the size of the fissioning nuclei compared with different model calculations. 
decreases with the size of the fissioning nuclei as expected for nuclei with lower fissility. An exception are the fissioning nuclei with $\mathrm{Z}_{1}+\mathrm{Z}_{2}=83$ and $\mathrm{Z}_{1}+\mathrm{Z}_{2}=84$ which are produced in charge-exchange reactions with a very small probability [55]. In the lower panel we observe a clear increase of the width of the charge distribution of the final fragments when decreasing the size of the fissioning nuclei. This behavior is interpreted as an increase of the temperature at saddle with the initial excitation energy. In this figure the measurements are used to benchmark different model calculations in order to quantify the magnitude of the dissipation coefficient. In particular we couple two intranuclear cascade codes describing the first stage of the collision, INCL4.6 [56] and ISABEL [57], to the deexcitation code ABLA07 [58].

In intranuclear cascade codes, the collision of a high-energy projectile within a target nucleus initiates a succession of binary nucleon-nucleon collisions that may lead to the emission of pre-equilibrium nucleons. In INCL and ISABEL, nucleons are described as point-like particles, and as a continuous medium perturbed by the collisions induced by cascade particles, respectively. Nucleons in INCL are endowed within a realistic phase-space density (Woods-Saxon in configuration space, hard Fermi sphere in momentum space), while in ISABEL, both projectile and particle follow a folded Yukawa density distribution. In INCL, the excitation energy and the angular momentum induced by the intranuclear cascade are computed according to particle-hole excitations. The cascade stops self-consistently when the prefragment is assumed to be thermalized. In contrast, in ISABEL the cascade stops when the energy is below a specific cutoff energy. In both codes, the remaining excitation energy is distributed among all the nucleons.

The ABLA code describes the deexcitation of a thermalized compound nucleus by fission or by the emission of light charged particles, $\gamma$-rays, neutrons and intermediate-mass fragments (IMF) following Weisskopfs model [59]. The fission probability is computed according to a time-dependent fission width following the analytical description of the solutions of the corresponding Fokker-Plank equation proposed in Refs. [30,60,61]. The code also allows to evaluate the fission width according to the transition-state model of Bohr and Wheeler [4] or the time-independent formulation of Kramers [5]. Important parameters in the code are the level density parameters that are calculated according to the parametrization proposed by Ignatyuk [62] and angular momentum dependent fission barriers which are taken from Sierks finite-range liquid drop model [63].

The benchmarking of these model calculations presented in Fig. 1 shows the sensitivity of the partial fission cross sections and the width of the charge 

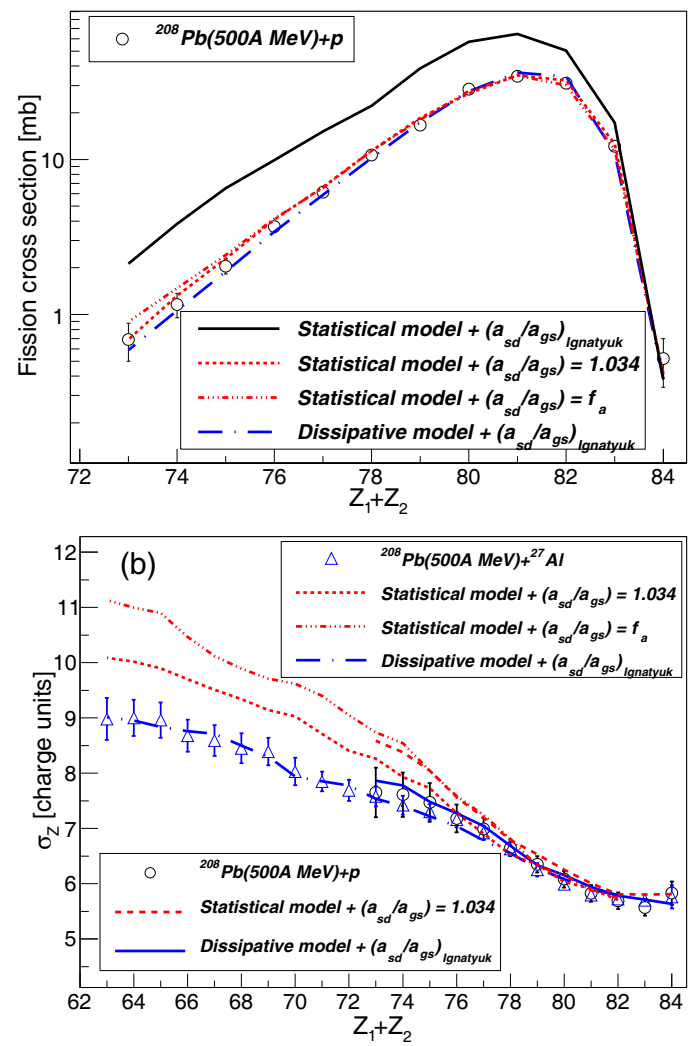

Figure 2: Upper panel: Fission cross section of the reaction ${ }^{208} \mathrm{~Pb}+\mathrm{p}$ at $500 \mathrm{~A} \mathrm{MeV}$ as a function of the atomic number of the fissioning nuclei (open circles). The lines represent model calculations with different descriptions of the fission decay width and the level density parameter (see text for details). Lower panel: Width of the atomic-number distribution of the final fission fragments measured in the reaction ${ }^{208} \mathrm{~Pb}+\mathrm{p}$ at $500 \mathrm{~A} \mathrm{MeV}$ as a function of the atomic number of the fissioning nuclei compared to the same model calculations shown in the upper panel. 
distribution of the final fragments to different intranuclear cascade codes as well as to the different descriptions of the fission decay width. It is clear that purely statistical calculations overestimate the partial fission cross sections and the width of the charge distributions. Only model calculations including a time-dependent description of the fission width with a viscosity parameter around $4.510^{21} \mathrm{~s}^{-1}$ describe correctly both observables. This magnitude of the dissipation parameter corresponds to a ground-to-saddle fission transient time of $1-510^{-21} \mathrm{~s}$. This result is in good agreement with others previously obtained using fusion-fission reactions [37-39,49]. Moreover, the fact that we are able to describe the data over a broad range of $\mathrm{Z}_{1}+\mathrm{Z}_{2}$ values with the same dissipation parameter would indicate that, contrary to some previous results, this parameter does not depend on temperature.

These observables have also been used in Ref. [50] to investigate the role of the description of the level densities at ground and saddle deformation in the fission process. The upper panel in Fig. 2 shows that different descriptions of the fission decay width can reproduce fission cross sections provided that specific values of the ratio of the level density parameter at ground and saddle deformation are chosen. This argument was used by the authors of Refs. $[51,52]$ to conclude that purely statistical descriptions of the fission decay width could describe the cross sections of fission reactions induced at high energies by using appropriate values for the level densities. We can then conclude that the theoretical description of the ground-to-saddle fission dynamics is a multiparametric problem that can only be solved by using several independent observables measured simultaneously.

\section{Saddle-to-scission dynamics}

Dissipative effects at large deformation has been traditionally investigated by measuring the prescission emission of neutrons [18,32, 33], GDR $\gamma$-rays $[17,34-36]$ or light-charged particles $[38,45]$. In many of these works fission cross sections measured in coincidence were used to determine the ground-to-saddle dissipation parameter. These results are in good agreement with the values obtained in spallation and fragmentation reactions discussed in the previous section $\beta_{\text {ground-saddle }} \approx 4-510^{21} \mathrm{~s}^{-1}$. Most of the experiments providing values of the dissipation parameter at large deformation using prescission GDR $\gamma$-rays $[35,36]$ and neutrons $[32,33]$ obtained values around $\beta_{\text {saddle-scission }} \approx 20-3010^{21} \mathrm{~s}^{-1}$. However, model calculations based on the Langevin equation obtained values for the dissipation parameter at large deformation around $\beta_{\text {saddle-scission }} \approx 610^{21} \mathrm{~s}^{-1}$ [64]. 

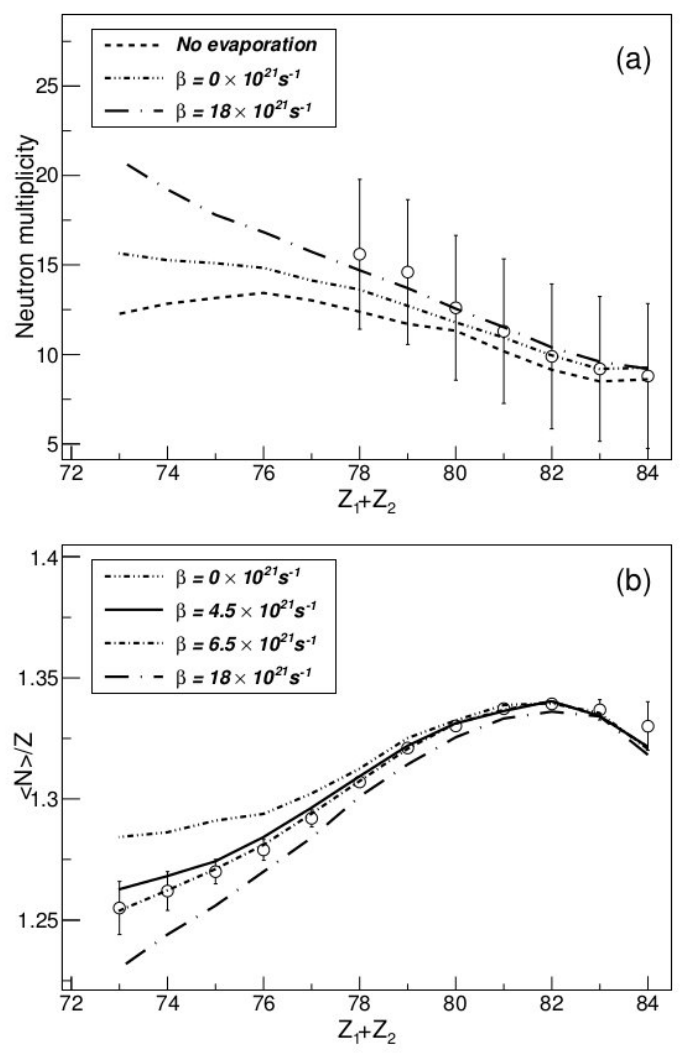

Figure 3: Upper panel: Prescission neutron multiplicities obtained for the reaction ${ }^{208} \mathrm{~Pb}+\mathrm{p}$ at $500 \mathrm{~A} \mathrm{MeV}$ as a function of the atomic number of the fissioning nuclei (open circles). The lines represent model calculations with different dissipation factors between saddle and scission. Lower panel: Neutron excess of the final fission fragments as a function of the atomic number of the fissioning nuclei also compared with model calculations with different dissipation factors between saddle and scission. 
Recently, an experiment to investigate dissipative effects at large deformation using the reaction ${ }^{208} \mathrm{~Pb}+\mathrm{p}$ at $500 \mathrm{~A} \mathrm{MeV}$ in inverse kinematics was conducted at GSI. The combination of a large acceptance dipole magnet with accurate tracking and time-of-flight detectors, and performant ionization chambers made it possible for the first time to identify in atomic and mass numbers both fission fragments and determine their velocities with high accuracy [23]. These measurements provided a precise characterization of the fissioning systems at scission and allowed to determine the prescission neutron multiplicities from the isotopic composition of the final fragments and their velocities using momentum conservation and the the kinematic model for fission from Ref. [65]. The results are shown in the upper panel of Fig. 3 as a function of the size of the fissioning nuclei.

The figure also depicts the result of several model calculations using different assumptions to describe the saddle-to-scission time. In particular calculations were run for the cases where no particle evaporation between saddle and scission is considered, the saddle-to-scission time is calculated from statistical considerations or this time is obtained with a dynamical calculation assuming a value of the dissipation parameter of $\beta_{\text {saddle-scission }}$ $=1810^{21} \mathrm{~s}^{-1}$ according to Ref. [66]. As can be observe, the rather large error bars associate to the multiplicity of prescission neutrons caused by the accuracy in the measurement of the velocity of the fission fragments does not allow to really benchmark those calculations.

An alternative was to use the neutron excess of the final fission fragments. Although the final value of the neutron excess of the fragments is defined by the neutron evaporation pre- and postscission, dissipative effects will only affect the prescision fraction. Moreover, this is an observable that it is determined with high accuracy in the mentioned experiment. In the lower panel of Fig. 3 we depict the neutron excess of the final fission fragments measured in the reaction ${ }^{208} \mathrm{~Pb}+\mathrm{p}$ at $500 \mathrm{~A} \mathrm{MeV}$ as a function of the size of the fissioning nuclei. As can be seen, the error bars are relatively smaller than in the case of the prescission neutron multiplicity shown in the upper panel of the figure.

In the lower panel of Fig. 3 we also show the results of the model calculations described above. This benchmarking clearly shows that the new observable is more sensitive to the different model calculations. Moreover, the evolution of the neutron excess over the complete range of size of the fissioning nuclei can be nicely describe with a value of the dissipation coefficient of $\beta_{\text {saddle-scission }}=6.5 \pm 2.010^{21} \mathrm{~s}^{-1}$. The difference with respect to previous measurements could be due to the low efficiency affecting prescission neutron and $\gamma$-ray measurements, and the limited sensitivity of these 
observables to the model calculations unless extremely accurate measurements are done.

Considering the final accuracy in the dissipation parameter at large deformation and the one obtained at small deformation $\beta_{\text {ground-saddle }}=$ $4.5 \pm 1.010^{21} \mathrm{~s}^{-1}$ it was concluded that both values are compatible, and no dependence of the dissipation parameter on deformation is then observed.

\section{Conclusions}

The investigation of the fission dynamics still represents a real challenge for physicists however, the last decade brought impressive progress in experimental and theoretical studies. From the experimental side one can highlight the use of inverse kinematics to better identify the fission fragments. Indeed, very recently it was possible for the first time to fully identify in atomic and mass number both fission fragments using inverse kinematics and a state-of-the-art detection setup.

The progress in the experimental techniques has also allowed to use fragmentation or spallation reactions to investigate fission at high excitation energy, low angular momenta and small shape distortion in the fissioning nuclei, fulfilling the optimal conditions for the study of the dynamics through dissipative effects in fission. These advanced experiments made also possible the use of new observables. Some of these observables could be measured simultaneously in the same experiment providing stringent constraints to the different parameters affecting the theoretical description of the fission process such as level densities at ground and saddle deformation and the dissipation parameter governing the coupling between intrinsic and collective degrees of freedom. Indeed, the magnitude and possible dependences of this parameter on deformation and temperature have been a subject of vivid debate.

Partial fission cross sections and the width of the charge distribution of the final fission fragments sorted as a function of the sum of the atomic numbers of the two final fragments, have been proven to be optimum observables to constraint the nuclear viscosity at small deformation. All studies suggest a value of the dissipation parameter at small deformation of $\beta_{\text {ground-saddle }}$ $=4.5 \pm 1.010^{21} \mathrm{~s}^{-1}$.

The recent isotopic identification of the final fission fragments gave access to a new and accurate observable to constraint the dissipation parameter at large deformation, the neutron excess of the final fragments. The description of this observable with model calculations is compatible with a value of the 
dissipation parameter from saddle to scission of $\beta_{\text {saddle-scission }}=6.5 \pm 2.0$ $10^{21} \mathrm{~s}^{-1}$. Considering the accuracy in both dissipation parameters it was concluded that they are compatible and then no evidence for a deformation dependence of this parameter is observed. Moreover, all observables are described over a broad range of sizes of the final fragments, very much correlated to the initial excitation energy, with the same values, therefore no dependence on temperature was observed.

\section{References}

[1] K.-H. Schmidt, J. Benlliure and A. Junghans, Nucl. Phys. A 693, 169 (2001).

[2] P. Fröbrich and I.I. Gontchar, Phys. Rep. 292, 131 (1998).

[3] S. Goriely, Eur. Phys. J. A 51, 22 (2015).

[4] N. Bohr and J.A. Wheeler, Phys. Rev. 56, 426 (1939).

[5] H.A. Kramers, Physica 7, 284 (1940).

[6] P. Grangé, L. Jun-Qing, and H. A. Weidenmüller, Phys. Rev. C 27, 2063 (1983).

[7] P. Möller and J. Randrup, Phys. Rev. C 91, 044316.

[8] P. Möller et al., Phys. Rev. C 91, 024310 (2015).

[9] J.D. MacDonnell et al., Phys. Rev. C 90, 021302 (2014).

[10] C. Simenel and A.S. Umar, Phys. Rev. C 89, 031601 (2014).

[11] C. Böckstiegel et al., Phys. Lett. B 398, 259 (1997).

[12] A.A Naqvi et al., Phys. Rev. C 34, 218 (1986).

[13] H. Nifenecker et al., Zeit für Phys. A 308, 39 (1982).

[14] K.-H. Schmidt and B. Jurado, Phys. Rev. lett. 104, 212501 (2010).

[15] B. Jurado and K.-H. Schmidt, J. Phys. G 42, 5 (2015).

[16] D. Jacquet and M. Morjean, Prog. Part. Phys. 63, 155 (2009).

[17] M. Thoennessen and G.F. Bertsch, Phys. Rev. Lett. 71, 4303 (1993). 
[18] D.J. Hinde et al., Phys. Rev. C 45, 1229 (1992).

[19] A.N. Andreyev et al., Phys. Rev. lett. 105, 252502 (2010).

[20] M. Bernas et al., Phys. Lett. B 331, 19 (1994).

[21] K.-H. Schmidt et al., 665, 221 (2000).

[22] M. Caamaño et al., Phys. Rev. C 92, 034606 (2015).

[23] J.L. Rodriguez-Sánchez et al., Phys. Rev. C 91, 064616 (2015).

[24] J. Benlliure et al., Nucl. Phys. A 683, 513 (2001).

[25] T. Enqvist et al., Nucl. Phys. A 686, 481 (2001).

[26] T. Enqvist et al., Nucl. Phys. A 703, 435 (2002).

[27] M. Bernas et al., Nucl. Phys. A 725, 213 (2003).

[28] T. Enqvist et al., Nucl. Phys. A 658, 47 (1999).

[29] J. Pereira et al., Phys. Rev. C 75, 014602 (2007).

[30] B. Jurado et al., Nucl. Phys. A 757, 329 (2005).

[31] W. Ye and J. Tian, Phys. Rev. C 91, 064603 (2015).

[32] D. Hilscher and H. Rossner, Ann. Phys. 17, 471 (1992).

[33] P. Fröbrich et al., Nucl. Phys. A 556, 281 (1993).

[34] D. J. Hofman et al., Phys. Rev. C 51, 2597 (1995).

[35] N.P. Shaw et al., Phys. Rev. C 61, 044612 (2000).

[36] I. Diosdegi et al., Phys. Rev. C 63, 014611 (2001).

[37] J.P. Lestone and S.G. McCalla, Phys. Rev. C 79, 044611 (2009).

[38] E. Vardaci et al., Phys. Rev. C 92, 034610 (2015).

[39] K.X. Jing et al., Phys. Lett. B 518, 221 (2001).

[40] J. Benlliure et al., Nucl. Phys. A 700, 469 (2002).

[41] J. Benlliure et al., Phys. Rev. C 74, 014609 (2006). 
[42] B. Lott et al., Phys. Rev. C 63, 034616 (2001).

[43] C. Schmitt et al., Phys. Rev. C 81, 064602 (2010).

[44] B. Jurado et al., Phys. Rev. Lett. 93, 072501 (2004).

[45] J.P. Lestone et al., Phys. Rev. Lett. 67, 1078 (1990).

[46] Y. Ayyad et al., Phys. Rev. C 89, 054610 (2014).

[47] J.L. Rodriguez-Sánchez et al., Phys. Rev. C 90, 064606 (2014).

[48] W. Ye, Phys. Rev. C 83, 044611 (2011).

[49] W. Ye and N. Wang, Phys. Rev. C 87, 014610 (2013).

[50] J.L. Rodriguez-Sánchez et al., Phys. Rev. C 92, 044612 (2015).

[51] D. Mancusi et al., Phys. Rev. C 82, 044610 (2010).

[52] S. Lo Meo et al., Nucl. Phys. A 933, 43 (2015).

[53] M.G. Itkis et al., Sov. J. Part. Nucl. 19, 301 (1988).

[54] Y. Ayyad et al., Phys. Rev. C 91, 034601 (2015).

[55] A. Kelic et al., Phys. Rev. C 70, 064608 (2004).

[56] A. Boudard et al., Phys. Rev. C 87, 014606 (2013).

[57] Y. Yariv and Z. Fraenkel, Phys. Rev. C 20, 2227 (1979).

[58] A. Kelic, M. V. Ricciardi, and K.-H. Schmidt, in Proceeding of Joint ICTP-IAEA Advanced Workshop on Model Codes for Spallation Reactions, ICTP Trieste, Italy, 48 February, 2008, edited by D. Filges, S. Leray, Y. Yariv, A. Mengoni, A. Stanculescu, and G. Mank (IAEA, Vienna, 2008), INDC(NDS)- 530, pp. 181221.

[59] V. Weisskopf, Phys. Rev. 52, 295 (1937).

[60] B. Jurado, K.-H. Schmidt, and J. Benlliure, Phys. Lett. B 533, 186 (2003).

[61] B. Jurado, et al., Nucl. Phys. A 747, 14 (2005).

[62] A. V. Ignatyuk et al., Sov. J. Nucl. Phys. 21, 612 (1975). 
[63] A. J. Sierk, Phys. Rev. C 33, 2039 (1986).

[64] A.V. Karpov et al., Phys. Rev. C 63, 054610 (2001).

[65] B.D. Wilkins et al, Phys. Rev. C 14, 1832 (1976).

[66] H. Hofmann and J.R. Nix, Phys. Lett. B 122, 117 (1983). 